\title{
Nutritional Control of Cellular Form in Trigonopsis variabilis
}

\author{
By S. SF'N'THF,SHANMUGANATHAN* AND W. J. NICKERSON \\ Institute of Microbiology, Rutgers, The State Lniversity, \\ New Brunswick, Nere Jersey, U.S.A.
}

(Reccived 9 May 1961)

\begin{abstract}
SUMMARY
The cellular form of Trigonopsis zariabilis may be either triangular or ellipsoidal in outline. Development of form in this yeast is subject to nutritional control. Cell populations, almost cxclusively of triangular form, are obtained in defined media containing methionine, or other suitable methyl group donor. Cultures in defined media devoid of methyl group donor usually consist cxclusively of ellipsoidal forms.
\end{abstract}

\section{INTKOIDUCTION}

In studies on the biochemical bases of morphogenesis in micro-organisms which have been conducted in this laboratory, ycast $\leftrightarrow$ filament transformation in Candida albicans has been investigated in detail (Nickerson, 1954; Falcone \& Nickerson, 1959), and filament $\leftrightarrow$ ycast conversion in Mucor ronxii has been explored (Bartnicki-Garcia \& Nickerson, 1959). Nore recently, an intriguing 'triangular' yeast (Trigonopsis) and 'lemon-shaped' yeasts (Klocekera) have excited our curiosity. We have inquired into molecular bases of form development in the ellipsoidal $\leftrightarrow$, triangular transformation of 'Trigonopsis, and present our findings on experimental control of this transformation. To designate a remarkable 'triangular' yeast Schachner (1929) proposed the generic name Trigonopsis; to stress his observation that cell populations of this organism consisted of both triangular and ellipsoidal forms, he selected variabilis as the specific designation. The unusual morphology of this yeast poses an intriguing problem in respect to its morphogenesis. The cellular envelope determines cellular form in a yeast; what differences in wall composition could result in abrupt changes such that an ellipsoidal form could develop a triangular bud? What arrangement of polymeric components in a wall fabric would form a triangular lozenge? Early in the course of the present work it was found that development of cellular form in Trigonopsis variabilis is subject to nutritional control. Nutrients that constitute donors of active methyl groups, and thus promote phospholipid synthesis, were found to foster the development of populations consisting exclusively of triangular forms. Conversely, cultures in defined media devoid of methyl donors were observed to consist exclusively of ellipsoidal forms of lower phospholipid content. 'To account for the molecular basis of form in $T$. variabilis, the composition and structure of isolated cell walls was

* Present address : Medical Research Institute, Colombo 8, Ceylon. 
examined. $\Lambda$ rchitectural and physical properties of polymeric components of the wall fabric were examined because of their role as ultimate detcrminants of cellular form. The present paper is devoted to the nutritional control of morphogenesis in T. variabilis.

\section{METHODS}

Microbiological methods. The organism used in this work was Trigonopsis variabilis Schachner (strain CBS 1040) obtained from the Centraalbureau voor Schimmclcultures, Yeast Division, Delft, Holland. Cultures were grown on a defined medium which contained (all quantities $/ 100 \mathrm{ml}$.): glucose, $2 \mathrm{~g}$.; $\mathrm{KH}_{2} \mathrm{PO}_{4}, 0.4 \mathrm{~g}$; $\mathrm{HgSO}_{4}$. $7 \mathrm{H}_{2} \mathrm{O}, 0 \cdot 1 \mathrm{~g} . ;\left(\mathrm{NH}_{4}\right)_{2} \mathrm{SO}_{4}, 0.2 \mathrm{~g} . ; \mathrm{CaCl}_{2}, 0.05 \mathrm{~g} . ; \mathrm{H}_{3} \mathrm{BO}_{3}, 10 \mathrm{mg} . ;$ ammonium mulybdate, $4 \mathrm{mg}$; $\mathrm{MnSO}_{4} .4 \mathrm{H}_{2} \mathrm{O}, 4 \mathrm{mg}$.; $/ \mathrm{nSO}_{4} .7 \mathrm{H}_{2} \mathrm{O}, 4 \mathrm{mg}$; $\mathrm{CuSO}_{4} .5 \mathrm{H}_{2} \mathrm{O}$, $4.5 \mathrm{mg}$; $\mathrm{FeSO}_{4} .7 \mathrm{H}_{2} \mathrm{O}, 2.5 \mathrm{mg}$. In preliminary studies, before the vitamin requircments of the yeast were ascertained, a supplement consisting of the following nutrilites was included : $400 \mu \mathrm{g}$. each of calcium ( + ) pantothenate, nicotinic acid, pyridoxine. HC] and thiamine. $\mathrm{HCl} ; 200 \mu \mathrm{g}$. each of folic acid, inositol, riboflavin and $p$-aminobenzoic acid; $2 \mu$ r. of biotin. The medium was adjusted to pII $6 \cdot 0$, and sterilized by autoclaving at $115^{\circ}$ for $20 \mathrm{~min}$. (Ylucose was sterilized separately by autoclaving. For studies of vitamin requirements, nutrilite solutions were sterilized by filtration.

Cultures were grown in conical flasks of a capacity two and a half times the volume of medium used. The inoculum was $0.1 \mathrm{ml}$. of a washed suspension of organism (optical density $\mathbf{0} \cdot \mathbf{2 0 0}$, as determined in a Klett-Summerson photo-electric colorimeter with filter No. 54) obtained from a $72 \mathrm{hr}$. liquid eulture that had been grown in the above medium. After inoculation, cultures were incubated at $28^{\circ}$ for $\tau 0-72 \mathrm{hr}$. on a rotatory shaker which was set at $250 \mathrm{rev} . / \mathrm{min}$. Stock cultures were maintained on slopes of the above medium solidified with $2 \%(w / v)$ agar (Difeo). When the vitamin requirement studies were completed, the standard medium was modified to include only two vitamins, namely, biotin $(2 \mu \mathrm{g} . / 100 \mathrm{ml}$.) and thiamine $\mathrm{HCl}(10 \mu \mathrm{g} . / 100 \mathrm{ml}$.$) . After ascertaining the influence of nitrogen source on$ development of cellular form, the 'basal medium' was defined to contain: glucose. salts, biotin and thiamine.

Measurement of groweth. Unless stated to the contrary, all growth measurements were made at the end of incubation for $72 \mathrm{hr}$. at $28^{\circ}$, and are expressed in terms of mg. dry weight organism per litre. Dry weight was determined by reference to a standard graph relating optical density to dry weight. Optical density was determined turbidimetrically with a Klett-Summerson photo-electric colorimeter, with filter No. 54, and uninoculated medium as reference.

Counts of organisms. Dry smears of cultures were made and stained negatively with nigrosin $(0 \cdot 1 \%, w / v)$ to reveal distinctly the outline of the organisms; counts were made of at least 250 organisms.

Reagents. The purity of amino acids, obtained from commercial sources, was ascertained by paper chromatography (Woiwood, 1949). The isomeric forms of compounds used are indicated in the text. DL-Methionine was purchased from Sigma Chemical Co., St Louis, and was recrystallized three times from hot water until chromatographically pure. D- and t-methionine, DI-homocysteine and DL-homocystine were obtained from Mann Rescarch Laboratories, New York, as chromatographically pure preparations. Wthionine and choline were obtained from 
Nutritional Biochemical Corporation, Cleveland, Ohio, U.S.A.; inositol from Corn Products Co., 17 Battery Place, New York; and glyceromono-oleate from Glyco l'roducts Co., Inc., Brooklyn 2, New York. 'This latter compound was free from any trace of biotin when estimated by the chromatographic method of Nickerson (1961) with Candida albicans 582 as test organism. Oleic acid was obtained from the California Corporation for Biochemical Research, Ios Angeles, California. Lecithin (soya bean; Nutritional Biochemicals Corp., Cleveland, Ohio) was purified by treatment with acetone. The pyrimidinc (1-amino-2-methyl-pyrimidyl-5-methylsulphonic: acid) and thiazole (4-methyl-5- $\beta$-hydroxyl thiazole) moieties of thiamine were prepared from thiamine by the method of Williams, Waterman, Keresztesy \& Buchman (1935). Preparations thus obtained were free from thiamine when tested by the thiochrome method (IIennessy \& Cereecdo, 1939). Glucose oxidase (Glucostat) was obtained from Worthington Biochemical Corporation, Freehold, N.J., U.S.A.

Solutions. Solutions of glyceromono-oleate and oleic acid were prepared in ethanol, sterilized by filtration and added aseptically to culture medium. Homocystine and homocysteine were dissolved in water immediately before use and sterilized by filtration.

Purification of agar. Difco Bacto agar (50 g.) was suspended in $500 \mathrm{ml}$. distilled water, stirred for $15 \mathrm{~min}$. and filtered; this treatment was effected twice. The washed agar was then shaken in $200 \mathrm{ml}$, of $95 \%(\mathrm{v} / \mathrm{v})$ ethanol in water and filtered. The residue was resuspended in $500 \mathrm{ml}$. distilled water, filtered, and washed again with $200 \mathrm{ml}$. of $95 \%(\mathrm{v} / \mathrm{v})$ ethanol in water. Material thus obtained was allowed to dry overnight. The agar was scraped from the filter and stirred with aqueous pyridine $(100 \mathrm{ml}$. redistilled pyridine $+350 \mathrm{ml}$. water $)$ for 15 min. and filtered; this process was repeated twice. Finally, the material was washed with ethanol, then acetone and dried. The material thus obtained was free from thiamine and biotin; Trigonopsis variabilis did not grow when these two vitamins were omitted from growth medium solidified with this washed agar.

Preparation of silica gel plates. 'To defined medium containing either $\left(\mathrm{NII}_{4}\right)_{2} \mathrm{SO}_{4}$ or DL-methionine as nitrogen source, sufficient silica gel was added to give a final concentration of $5 \%(\mathrm{w} / \mathrm{v})$, and adjusted to $\mathrm{pH} 5.9$ with dilute $\mathrm{NaOH}$. This mixture was poured into plates and autoclaved at $120^{\circ}$ for $20 \mathrm{~min}$. (see Falcone \& Nickerson, 1958). Plates were seeded with a washed suspension of organisms which had been grown in $\left(\mathrm{NH}_{4}\right\rangle_{2} \mathrm{SO}_{4}$ basal medium containing thiamine $(10 \mu \mathrm{g} . / 100 \mathrm{ml}$. $)$. Plates wore incubated at $28^{\circ}$ in a water-saturated atmosphere to prevent rapid drying of the medium.

\section{RESULTS}

Nutritional requirements of Trigonopsis variabilis

Growth with different carbohylirate sources. Carbohydrate requirements of Trigonopsis variabilis werc examined by Lodder \& Kreger-van Rij (1952) in their taxonomic study of yeasts; they reported that this organism grew only with glucose or galactose as sources of carbohydratc. Our results (Table 1) are in ayrcement, except that we also observed moderate growth with sucrose; the sucrose used was ascertained to be free from glucose, as judged by the glucose oxidase method. After incubation for $72 \mathrm{hr}$., growth with glucose, fructose or mannose was in the ratio $1 \cdot 6: 1 \cdot 6: 1 \cdot 0$. The effect of glucose concentration on the rate of growth was also 
examined. Neither rate of growth nor amount of growth after $72 \mathrm{hr}$. changed appreciably above a glucose concentration of $2 \%(\mathrm{w} / \mathrm{v})$.

Growth in presence of different amino acids. There are no available data about the ability of this yeast to utilize any nitrogen source other than $\left(\mathrm{NH}_{4}\right)_{2} \mathrm{SO}_{4}$. Of thirteen amino acids tested as sole source of nitrogen (Table 2), eleven supported growth (DL-isoleucine and L-valine were not suitable as sole sources of nitrogen). Growth was most marked with alanine or proline as nitrogen source (900 $\mathrm{mg}$. dry wt.(l.); asparagine and leucine gave an intermediate response, whilst with

\section{Table 1. Growth of Trigonopsis variabilis with different carbohydrate sources}

Medium, with vitamin supplement, as described in text; carbohydrate concentration, $2 \%(w / v)$; total volume, $50 \mathrm{ml}$. 'The values (mg. dry wt./l.) are the averages of triplicate cultures from two sets of experiments.

\begin{tabular}{|c|c|c|c|c|c|}
\hline & \multicolumn{5}{|c|}{ Incubation period (hr.) } \\
\hline & 24 & 44 & 56 & 68 & 72 \\
\hline & \multicolumn{5}{|c|}{ Growth (mg. dry wt. organism/l. culture) } \\
\hline Carbohydrate & & & & & \\
\hline None & 0 & 0 & 0 & 0 & 0 \\
\hline Glucose & 4 & 126 & $\$ 14$ & 636 & 655 \\
\hline Galactose & 4 & 84 & $2 \tau \theta$ & 536 & 580 \\
\hline Sucrose & 4 & 44 & 84 & 88 & 88 \\
\hline Maltose & 2 & 2 & 2 & 3 & 3 \\
\hline Lactose & 2 & 2 & 2 & 8 & 2 \\
\hline
\end{tabular}

Table 2. Growth of Trigonopsis variabilis with different amino acids as sources of nitrogen

Conditions as described in Table 1. The nitrogen sources were supplied at a level of $200 \mathrm{mg}$. I. - or $400 \mathrm{mg}$. vL-form per $100 \mathrm{ml}$. The values ( $\mathrm{mg}$. dry wt./l.) are the averages of duplicate cultures from two sets of experiments.

Incubation period $(\mathbf{h r}$.)

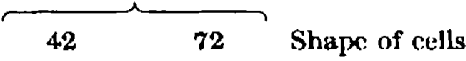

Growth (ing. dry wt./l.) after $72 \mathrm{hr}$.

\begin{tabular}{|c|c|c|c|}
\hline Nitrogen source & & & incubation \\
\hline None & o & 0 & - \\
\hline$\left(\mathrm{NH}_{4}\right)_{2} \mathrm{SO}_{4}$ & 95 & 700 & e \\
\hline I,-aspartic acid & 24 & 128 & e \\
\hline L-asparagine & 40 & 648 & $\mathbf{e}$ \\
\hline DL. $-x$-alanine & 210 & 970 & $\triangle$ and $\mathbf{e}$ \\
\hline L-cysteine & 24 & 58 & e \\
\hline Na-glutamate & 14 & 116 & e \\
\hline L-histidine & 8 & 260 & e \\
\hline I.-leucine & 48 & 695 & c \\
\hline DL-isoleucine & 2 & 10 & e \\
\hline I) -methionine & 16 & 268 & $\triangle$ \\
\hline L-proline & 210 & 920 & $\triangle$ and $\mathrm{e}$ \\
\hline vL-phenylalanine & 8 & 90 & e \\
\hline DL-threonine & 6 & 75 & e \\
\hline$\left(\mathrm{NHI}_{4}\right)_{2} \mathrm{SO}_{4}+$ vL-ulanine* & 150 & 920 & e \\
\hline L-valine & 10 & 30 & $\mathrm{e}$ \\
\hline
\end{tabular}

* $100 \mathrm{mg}$. $\left(\mathrm{NH}_{4}\right)_{2} \mathrm{SO}_{4}+100 \mathrm{mg}$. DL-alanine/100 $\mathrm{ml}$.

$\dagger \mathrm{e}=$ ellipsoidal; $\Delta=$ triangular. 
aspartate, glutamate, histidine or methionine, growth was less than one-seventh of that obtained with alanine or $\left(\mathrm{NH}_{4}\right)_{2} \mathrm{SO}_{4}$.

Morphology of populations in amino acid media. Cellular morphology was observed to vary with the nitrogen source supplied. With alanine and proline, the population was a mixture of triangular and ellipsoidal forms, whilst with ammonium sulphate and the remaining amino acids (except methionine) more than $97 \%$ of the organisms were ellipsoidal. In contrast, more than $97 \%$ of the organisms

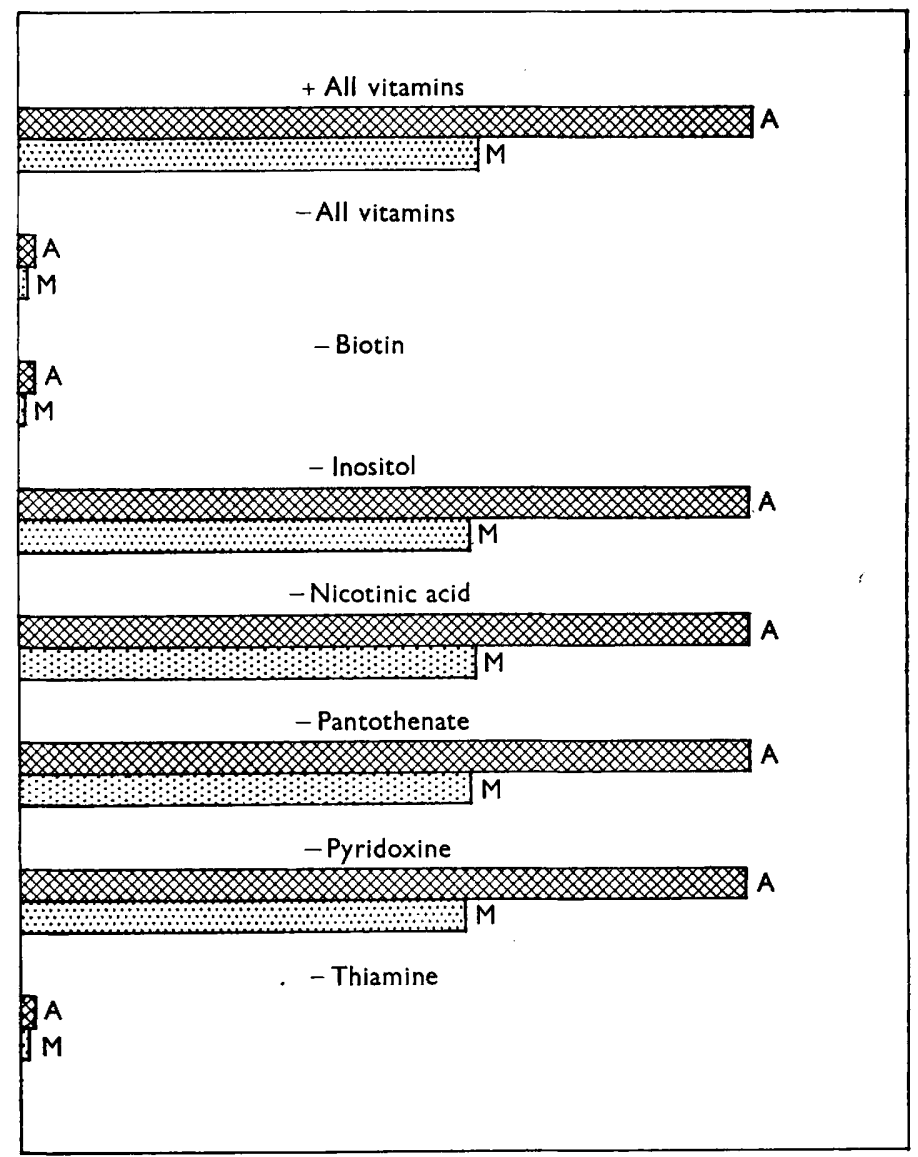

Relative growth response

Fig. 1. Vitamin requirements of Trigonopsis variabilis with $\left(\mathrm{NH}_{4}\right)_{2} \mathrm{SO}_{4}$ or DL-methionine as sole source of nitrogen. Basal medium and growth conditions as described in text. Incubation period: $72 \mathrm{hr}$. $\mathrm{A}=\left(\mathrm{NH}_{4}\right)_{2} \mathrm{SO}_{4}$ as sole source of nitrogen; $\mathrm{M}=$ DL-methionine as sole source of nitrogen.

were of triangular outline when methionine was the nitrogen source (see Table 2). It should be emphasized that organisms in cultures incubated for less than $48 \mathrm{hr}$. were all ellipsoidal, irrespective of the nitrogen source.

Vitamin requirements. Growth factor requirements of Trigonopsis variabilis were studied with methionine or $\left(\mathrm{NH}_{4}\right)_{2} \mathrm{SO}_{4}$ as nitrogen sources. In Fig. 1, it is shown that this yeast required only biotin and thiamine for optimal growth. Addition of 
other vitamins, either singly or in mixtures, did not produce further increase in rate or amount of growtl. The potential usefulness of this yeast for the assay of thiamine was explored. The response to thiamine was lincar over a range from about 0 to $7 \mu \mathrm{g} . j 100 \mathrm{ml}$. (Fig. 2), despite the fact that growth with ammonium sulphate was almost double that with methionine as source of nitrogen. This finding serves to emphasize that the source of nitrogen cannot be neglected in the design of basal media for vitamin assays.

When supplied with the thiazole moiety of thiamine, Trigonnpsis variabilis grew substantially as well as when supplied with pre-formed thiamine (Table 3). Since

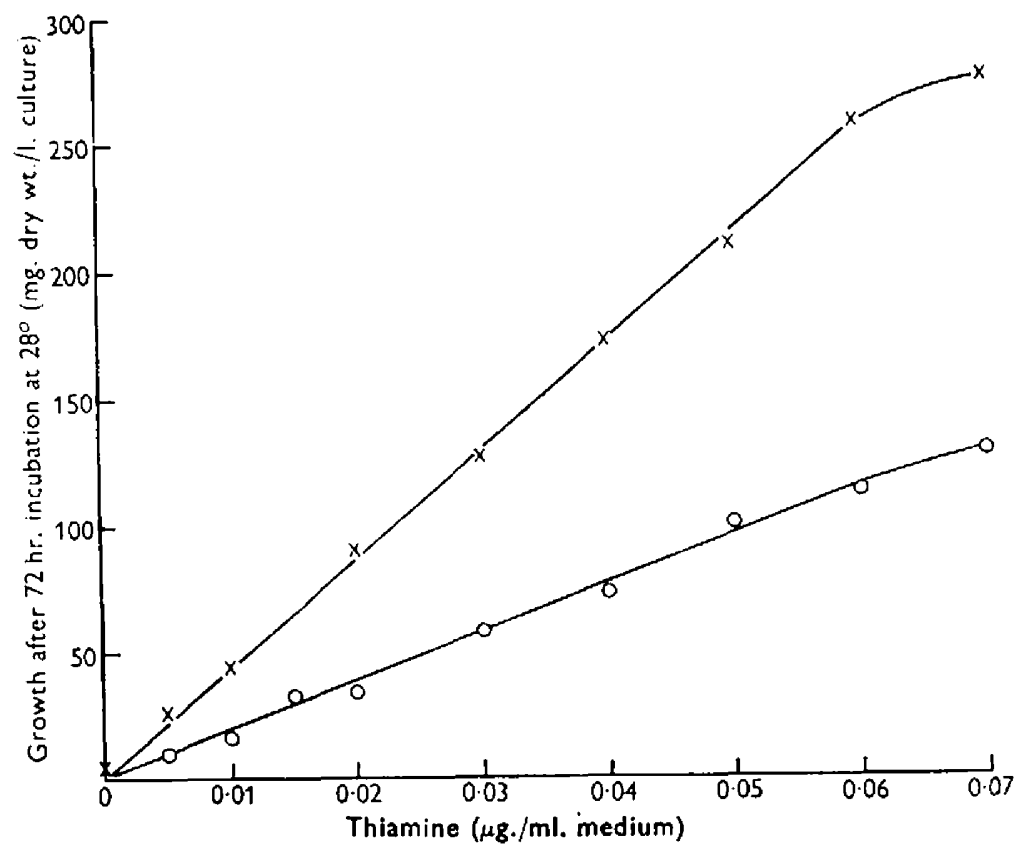

Fig. 2. Effect of thiamine concentration on the growth of Trigonopsis variabilis. $\times-\times$ $\left(\mathrm{NH}_{4}\right)_{2} \mathrm{SO}_{2}$ as $\mathrm{N}$ source $(25 \mathrm{mg}$. $\mathrm{N} / 100 \mathrm{ml}$.); $\mathrm{O}-\mathrm{O}$ vL-methionine as $\mathrm{N}$ source $(25 \mathrm{mg}$. $\mathrm{N} / 100 \mathrm{ml}$.).

Table 3. Effect of pyrimidine and thiazole moieties of thiamine on the growth of Trigonopsis variabilis

Medium contained biotin $(\mathbf{2} \mu \mathrm{g} . / 100 \mathrm{ml}$.) in addition to thiamine moiety. The nitrogen source was supplitd at $25 \mathrm{mg}$. N/100 ml. The growth factors $(1 \mu \mathrm{g} . / \mathrm{ml}$.) were sterilized by filtration and added to the medium aseptically 'The values for amount of growth (nig. dry wt./1.) are the averages of duplicate cultures from two sets of experiments after incubation for 72 hr. at $28^{\circ}$.

None

Thiamine

Pyrimidine

Thiazole

Thiamine + pyrimidine

Thiazole + pyrimidine

$$
\underset{\text { medium }}{\left(\mathrm{NH}_{4}\right)_{2} \mathrm{SO}_{4}} \begin{gathered}
\text { DL-methionine } \\
\text { medium }
\end{gathered}
$$

growth (mg. dry wt./l.)

$\begin{array}{rr}70 & 0 \\ 760 & 480 \\ 6 & 6 \\ 700 & 400 \\ 700 & 400 \\ 700 & 400\end{array}$


the pyrimidine moiety used contained a sulphonic acid group, the possibility of its acting as an inhibitory agent was considered. However, mixtures of the pyrimidine, with thiamine and with the thiazole moiety, gave no evidence of such action. It may be concluded, therefore, that biotin and the thiazole moiety of thiamine are essential nutrient factors for $T$. variabilis.

Partial replacement of the biotin requirement. It was shown by Nickerson (1961) for a baker's yeast strain and for Candida albicans 582 (an organism which requires only biotin as a growth factor), that biotin was absolutely replaceable by glyceromono-oleate (GMO) at $30 \mathrm{mg} . / 1 .\left(8.5 \times 10^{-5} \mathrm{M}\right)$. Oleic acid was without such effect for these yeasts. Above $40 \mathrm{mg}$./l., GMO was toxic for both of these organisms. It was found that Trigonopsis variabilis could also utilize GMO to replace biotin as a growth factor. With GMO growth occurred to an extent of about $25 \%$ of that with biotin, and a pronounced lag was evident. When GMO was incorporated in the medium with biotin, a stimulation in the rate of growth was observed. At concentrations above $40 \mathrm{mg}$./l., GMO suppressed growth.

\section{Nutritional control of morphogenesis in Trigonopsis variabilis}

Growth with different isomers of methionine. In view of significant differences in form between organisms grown with methionine or with ammonium sulphate as sole source of nitrogen, further investigations with these nitrogen sources were made. As shown in Fig. 3, rates of growth of Trigonopsis variabilis with D- or DL-methionine were identical, and greater than that with $\mathrm{L}$-methionine. The $\mathrm{D}$ - and L-amino acids used for this study were tested for contamination with the other isomer by the use of D-amino acid oxidase (prepared from hog kidney cortex according to Meister, 1952). The isomers were thus found to be of greater than $99 \%$ purity. These observations suggest that under aerobic conditions $\mathrm{D}$-methionine is deaminated at a faster rate than the L-isomer, implying that different enzyme systems are involved. These, and related results obtained with cell-free systems, will be described in a subsequent paper.

Form of groroth of Trigonopsis variabilis in liquid cultures. On transfer from stock culture (yeast extract + glucose agar) to medium M (basal medium + DL-methionine), $87 \%$ triangular and $13 \%$ ellipsoidal forms were obtained after incubation for 3 days ('Table 4). Transfer from solid medium $M$ to liquid medium $A$ resulted in $\mathbf{3} \%$ triangular and $\mathbf{9 7} \%$ ellipsoidal, whereas transfer to liquid medium $\mathbf{M}$ yielded $\mathbf{9 5} \%$ triangular and $5 \%$ ellipsoidal. It is reasonably clear that development of triangular forms on methionine medium is attributable to nutritional control of form-development and not to selection from a mixed population. Repeated transfer from liquid medium $\mathbf{M}$ to $\mathbf{A}$, or transfer to medium $\mathbf{A}$ after many passages in liquid medium $\mathbf{M}$ always resulted in the prompt development of an exclusively ellipsoidal population. Conversely, transfer to liquid medium $M$ after many passages in medium A always resulted in the development of about $95 \%$ triangular forms after a culture had been incubated for $72 \mathrm{hr}$. In view of the incubation time in this case, a question as to the operation of a population-selection phenomenon naturally arises. As shown in Fig. 3, however, T. variabilis enters the logarithmic phase of growth in methionine liquid medium only after about $40 \mathrm{hr}$. of incubation. This result was obtained even on frequent and repeated passage in medium $M$. In medium $A$, the logarithmic phase of growth begins after about $28 \mathrm{hr}$. of incubation. With either medium the 


\section{S. SentheShanmuganathan and W. J. Nickerson}

logarithmic phase persists until about the 50th hr. After about $48 \mathrm{hr}$. of incubation in medium $M$ appreciable numbers of triangular forms are evident (40\% of the total growth has been achieved by this time), and after about $70 \mathrm{hr}$. of incubation about $95 \%$ of all organisms are triangular forms. Conversion of ellipsoidal forms to triangular forms occurs in the stationary phase; buds formed toward the end of the logarithmic phase are predominantly triangular. Repeated and frequent passage in

Table 4. Form of Trigonopsis variabilis on repeated transfer from solid to liquid medium containing either methionine $(M)$ or $\left(\mathrm{NH}_{4}\right)_{2} \mathrm{SO}_{4}(A)$ as sole source of nitrogen

Standard basal medium was used; agar medium solidified with vitamin-free agar $(2 \%, w / v)$. Nitrogen source added at $25 \mathrm{mg}$. N/100 ml. The percentage of triangular (T) and ellipsoidal (E) forms was calculated from a total count of 250 organisms.

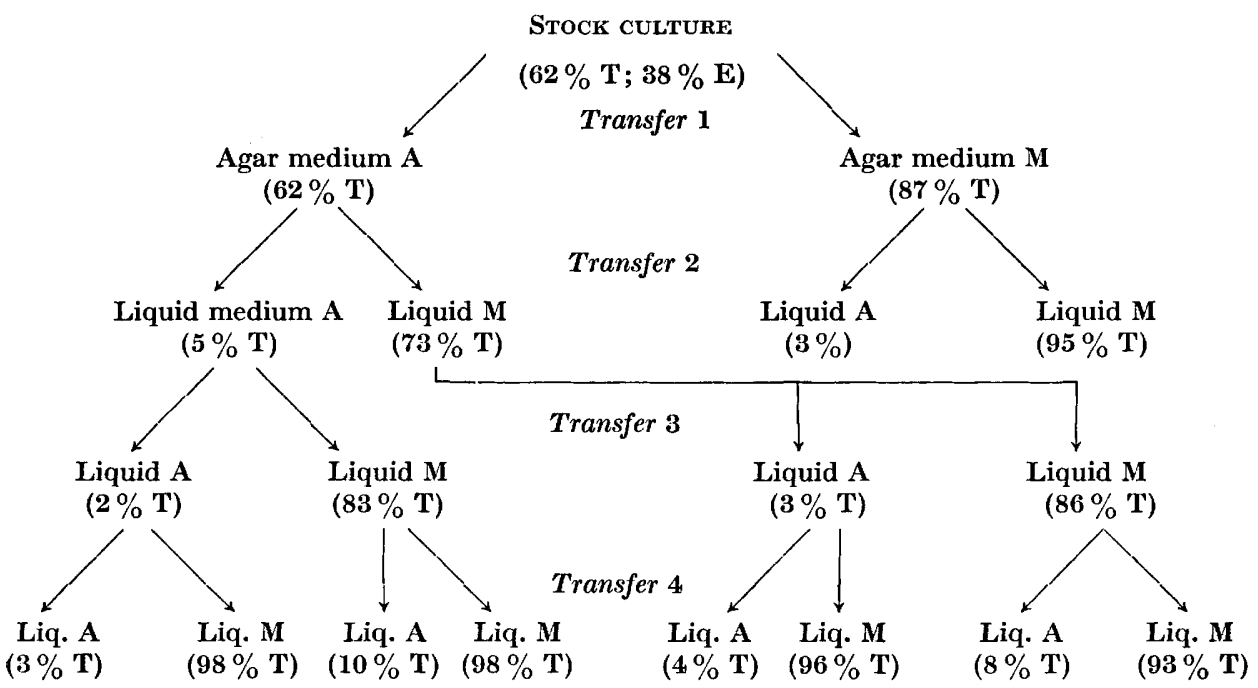

medium M does not alter the sequence of events just depicted. An inoculum comprised almost exclusively of triangular forms gives rise after a pronounced lag to ellipsoidal forms which, in turn, are converted into or give rise to triangular forms with onset of the stationary phase. Triangular organisms formed buds at the apices of the triangle; the budded young cells might be triangular or ellipsoidal. In many instances, ellipsoidal forms were found to bud and to give rise to triangular forms. The different forms of these organisms can be observed in the photomicrographs (Fig. 4).

Cultures on solidified media. With agar medium, irrespective of nitrogen source, the results were not consistent; sometimes as few as $75 \%$ triangular forms were obtained in medium M. On the other hand, the results obtained with liquid media were very uniform and variations were only about 5-7\%. Variability in results obtained with cultures on solid medium $\mathbf{A}$ or $\mathbf{M}$ might result from the presence of extraneous material in the agar, but experiments with purified agar yielded similarly variable results. Experiments were then made in shake cultures containing $\mathbf{0 . 3} \%$ $(\mathrm{w} / \mathrm{v})$ agar (Table 5). When agar was added to liquid medium $\mathrm{A}$, the proportion of triangular forms increased sharply from 5 to $66 \%$, whereas the addition of agar to medium $M$ resulted in a slight decrease in the proportion of triangular forms. The 
experiments just outlined were repeated in media solidified with silica gel, but not more than $88 \%$ triangular forms was obtained in medium $M$, whereas the proportion of triangular forms in medium $\mathrm{A}$ was of the order of $65 \%$ (Table 5). We have no explanation for this discrepancy between the results obtained with liquid and those on solidified or semi-solid media.

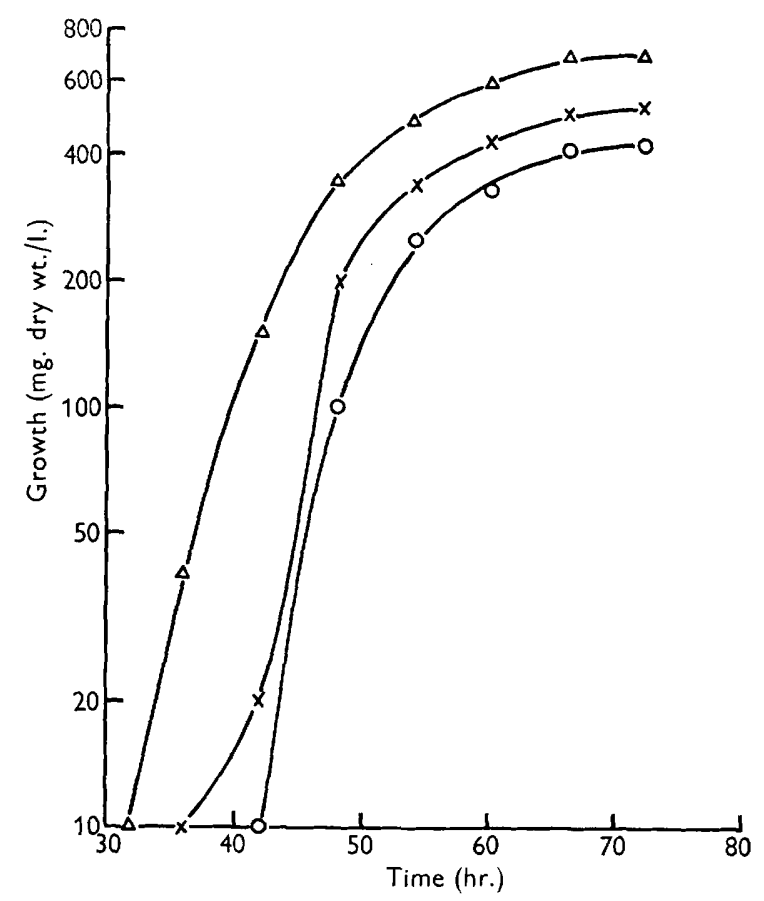

Fig. 3. Growth of Trigonopsis variabilis with D-, L-, or DL-methionine or $\left(\mathrm{NH}_{4}\right)_{2} \mathrm{SO}_{4}$ as sole source of nitrogen $(25 \mathrm{mg}$. N/100 ml.). Basal medium, as described in text, supplemented with biotin $\left(2 \mu \mathrm{g} . / 100 \mathrm{ml}\right.$.) and thiamine $(10 \mu \mathrm{g} . / 100 \mathrm{ml}.) . \Delta \cdots \Delta\left(\mathrm{NH}_{4}\right)_{2} \mathrm{SO}_{4}$; $\times \cdots \times \mathrm{D}$ - or DL-methionine; $0 \cdots \cdots \mathrm{L}$-methionine.

Table 5. Effect of solidifying agents on the morphology of

Trigonopsis variabilis

To the basal medium, agar was added (where indicated) to a final concentration of $0 \cdot 3 \%(w / v)$. Silica gel plates were prepared as described in text. Inocula consisted of organisms grown in liquid medium with $\left(\mathrm{NH}_{4}\right)_{2} \mathrm{SO}_{4}$ as nitrogen source. All experiments were performed twice, in duplicate.

\begin{tabular}{ccc} 
& \multicolumn{2}{c}{ Form of organisms (\%) } \\
Additions to basal medium & $\overbrace{\text { Triangular Ellipsoidal }}$ \\
Expt. 1 (semi-solid agar) & 5 & 95 \\
$\left(\mathrm{NH}_{4}\right)_{2} \mathrm{SO}_{4}$ & 66 & 34 \\
$\left(\mathrm{NH}_{4}\right)_{2} \mathrm{SO}_{4}+$ agar & 90 & 10 \\
DL-Methionine & 75 & 25 \\
DL-Methionine + agar & & \\
Expt. 2 (silica gel) & 65 & 35 \\
(NH $)_{2} \mathrm{SO}_{4}(10 \mathrm{mg})$. & 68 & 32 \\
(NH $)_{2} \mathrm{SO}_{4}(25 \mathrm{mg})$. & 88 & 12 \\
DL-Methionine (10 mg.) & 82 & 18 \\
DL-Methionine (25 mg.) &
\end{tabular}


Effect of transmethylating agents on morphology. Several compounds, such as methionine, choline, betaine or dimethyl- $\beta$-propiothetin can serve as methyl donors. Stekol et al. (1953) reported that synthesis of choline (a component of phospholipids) from dimethylaminoethanol involves a transmethylation reaction in which methionine supplies a preformed methyl group. Thus, in promoting morphological change in Trigonopsis variabilis, methionine might supply preformed methyl groups for the synthesis of choline or choline-containing compounds such as lecithin. If so, the use of homocysteine (the 'demethylated' precursor of methionine) or homocystine, as sole source of nitrogen, should result in predominantly ellipsoidal populations.

Table 6. Effect of choline, inositol and folic acid on growth and form of Trigonopsis variabilis with precursors or antagonist of methionine

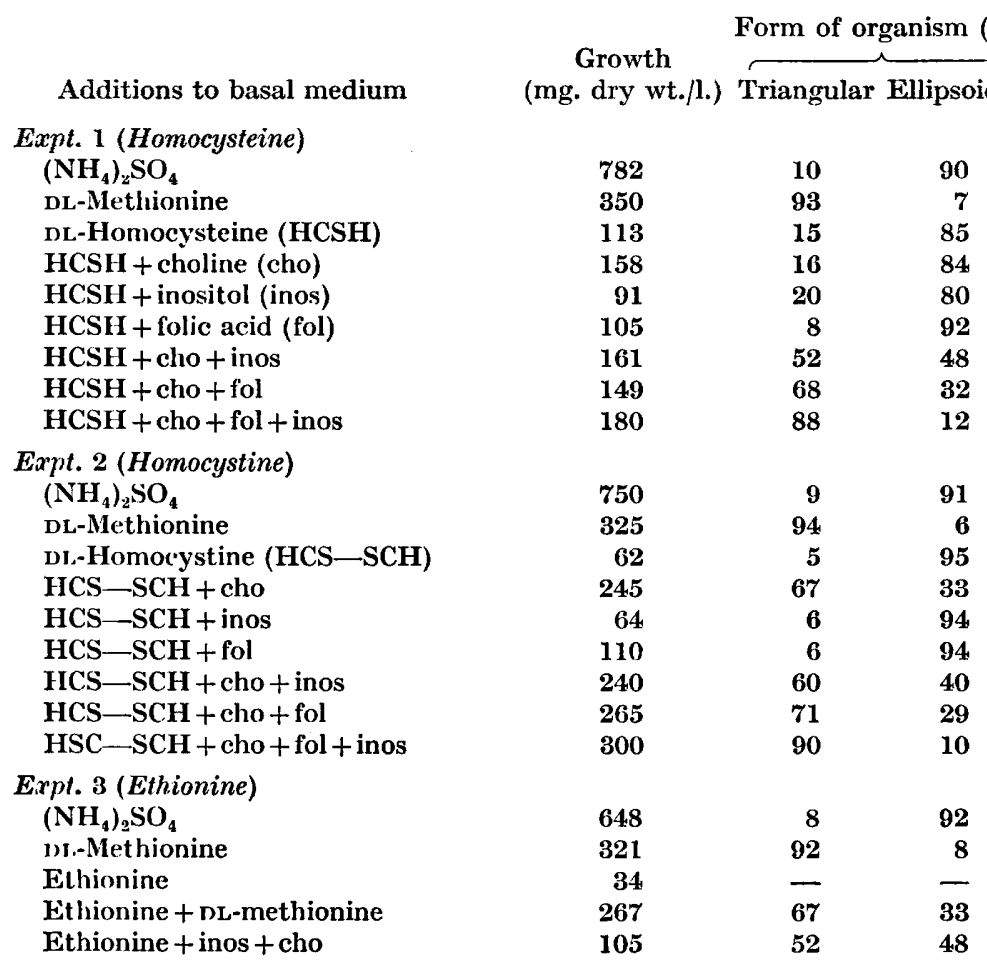

With homocysteine as nitrogen source (Table 6 ) there developed $85 \%$ ellipsoidal forms and only $15 \%$ triangular forms. The addition of choline did not alter this result, but when folic acid was also added, the proportion of triangular forms increased to about $65 \%$. Although the addition of inositol did not produce any further increase in the relative proportion of triangular forms, the mixture of folic acid, choline and inositol with homocysteine prompted the formation of triangular forms to an extent almost equal to that obtained with methionine alone. The results with homocystine as nitrogen source were similar to those just detailed, except that a noticeable increase in the proportion of triangular forms was obtained on supplementation with choline $(67 \%$ as compared to $5 \%$ triangular when choline was omitted). Choline also stimulated growth in the presence of homocystine. 
Ethionine (an antagonist for methionine) was not effective in supporting growth when supplied as sole source of nitrogen (Table 6, Expt. 3). When ethionine and methionine were added in equal weights, not only was growth inhibited by $20 \%$ but the 'methionine effect' on the morphology was inhibited by about $25 \%$.

Effect of phospholipid moieties on morphology. The addition of inositol to medium A stimulated development of triangular forms; this may be taken as further evidence that form-development in Trigonopsis variabilis is dependent upon synthesis of lipids and phosphatides. To examine this hypothesis, experiments were conducted with components of phospholipids, e.g. choline, glycerol and glycerol esters. The results (Table 7) show that the addition of choline and glycerol to a medium containing $\left(\mathrm{NH}_{4}\right)_{2} \mathrm{SO}_{4}$ as nitrogen source increased the proportion of triangular forms $(74 \%$ as compared to $10 \%$ ), and glyceromono-oleate supported $22 \%$ triangular forms. Although lecithin was found to inhibit growth, the two forms were found in equal numbers. The free and bound lipid contents of whole organisms and isolated walls of triangular forms were found to be substantially higher than in ellipsoidal forms (SentheShanmuganathan \& Nickerson, $1962 b$ ).

\section{Table 7. Effect of phospholipid components on the growth and form of Trigonopsis variabilis}

To the standard basal medium containing $\left(\mathrm{NH}_{4}\right)_{2} \mathrm{SO}_{4}$ as sole source of nitrogen, the following, where noted, were added (per $100 \mathrm{ml}$.): choline, $20 \mathrm{mg}$.; glycerol, 9.2 mg.; glyceromono-oleate (GMO), $3 \cdot 2 \mathrm{mg}$; lecithin, $2 \mathrm{mg}$. The inoculum consisted of washed suspension of organisms grown with $\left(\mathrm{NH}_{4}\right)_{2} \mathrm{SO}_{4}$ as nitrogen source.

$\begin{array}{lccc}\begin{array}{c}\text { Additions to } \\ \text { basal medium }\end{array} & \begin{array}{c}\text { Growth } \\ \text { (mg. dry wt./1.) }\end{array} & \overbrace{\text { Triangular Ellipsoidal }}^{\text {Form of organism (\%) }} \\ \text { None } & 453 & 10 & 90 \\ \text { Choline } & 445 & 33 & 67 \\ \text { Choline + glycerol } & 540 & 74 & \mathbf{2 6} \\ \text { GMO } & 510 & \mathbf{2 2} & \mathbf{7 8} \\ \text { Choline + lecithin } & 177 & 50 & \mathbf{5 0}\end{array}$

\section{DISCUSSION}

During studies on nutritional requirements of Trigonopsis variabilis it was observed that organisms obtained with methionine as sole source of nitrogen were mainly triangular, whereas those obtained with $\left(\mathrm{NH}_{4}\right)_{2} \mathrm{SO}_{4}$ were mainly ellipsoidal. This phenomenon of dimorphism was very marked in liquid cultures. An attempt was made to elucidate the manner in which nutritional control of form-development in this yeast is mediated biochemically. In addition to its appearance in most proteins, methionine (an indispensable component of the diet of higher animals) has been implicated in a wide variety of biosynthetic reactions : formation of lignin, nicotine, spermidine, creatine, adrenalin, anserine and cysteine (see Fruton \& Simmons, 1958). All but the latter of these involvements are improbable in the present connexion. Methionine is known to be a member of the 'aspartate family' of amino acids in Escherichia coli (Roberts \& Abelson, 1953) and in Neurospora crassa (Abelson \& Vogel, 1955). Furthermore, Rose \& Wixom (1955) have shown that a large part of the dietary requirement of man for methionine may be met by 
feeding cystine (cysteine). Since other members of the aspartate family, as well as cysteine (cystine), are devoid of ability to stimulate the production of triangular forms, we may dismiss the many involvements growing out of these familial associations in our search for the modus operandi of methionine on morphogenesis of $T$. variabilis. Studies with homocysteine (homocystine), the methyl-free precursor of methionine, provided indications that the morphogenetic action of methionine resides in its operation as a methyl donor. Methionine was found to be replaceable either by choline + inositol (with ammonium sulphate as the nitrogen source) or by choline alone (with homocysteine as nitrogen source). These, and other data from nutritional studies, point strongly to an inter-relationship between phospholipid synthesis and the morphology of this yeast.

The two forms of Trigonopsis variabilis have been termed either 'triangular' or 'ellipsoidal' in outline. Naturally, a question arises as to the three-dimensional aspect of the 'triangular' forms. As shown in Pl. 1, fig. 2 (and especially, as shown in Fig. 1 of the following paper (SentheShanmuganathan \& Nickerson, $1962 b$ ), this form is triangular in plane view and lozenge-shaped in cross-section; it is not tetrahedral nor pyramidal in three-dimensional aspect. The geometrical implications of transformation from an ellipsoidal pattern (prolate spheroid in three dimensions) to a triangular lozenge (three-dimensional aspect) resolve to those concerned with continuous deformation of a prolate spheroid which, without break in surface continuity, i.e. continuously differentiable deformation, lead to a triangular lozenge shape. In this transition, obviously, the surface/volume ratio is increased; simultaneously, surface tension at the organism/medium interface is decreased dramatically. Since measurements of gas transport (see SentheShanmuganathan \& Nickerson, $1962 c$ ) are only modestly in favour of the triangular form, we are inclined to attach only secondary significance to alteration in surface/volume ratios. Moreover, in view of the nature of biosyntheses favoured by a methyl-donor environment and the effect thereof on cellular lipid-composition (SentheShanmuganathan \& Nickerson, to be published), we feel that considerable importance must be attached to synthesis of polymeric components tending to minimize surface tension at the cell/medium interface. We may here be dealing with a biological example of the classical problem of Plateau (see Radó, 1951): ' to determine the surface of least area with a given boundary' (Bell, 1940). Such problems are easily visualized (as Plateau demonstrated) as soap films which span wire models of the boundary. Here, however, we are dealing with an underlying architecture that determines boundary shape and, hence, the surface of minimal area which encompasses the boundary. We shall defer further consideration of molecular bases of form development in this organism to the third paper in this series, where we shall return to a consideration of factors that determine the triangular boundary of this organism.

Supported in part by grants from the National Institutes of Health, U.S. Public Health Service. One of us (S.S.) was Pfizer Postdoctoral Fellow in Microbiology, on leave of absence from the Medical Research Institute, Colombo 8, Ceylon, when this work was done. 



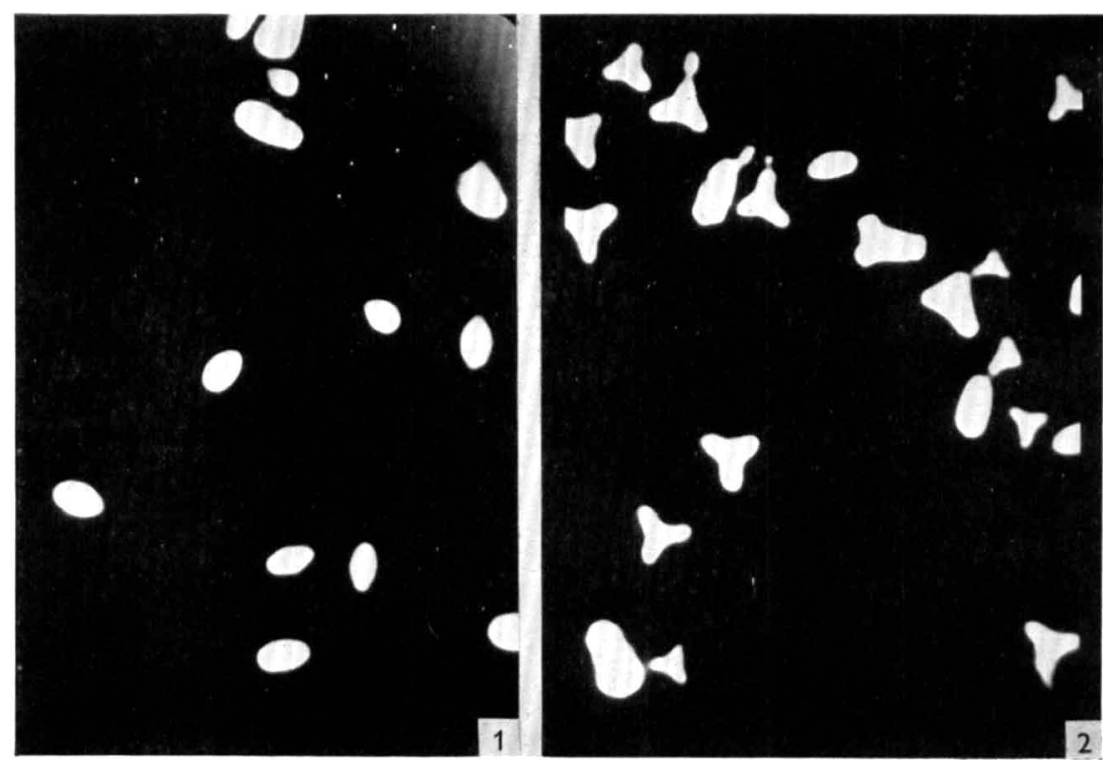




\section{REFERENCES}

Abelson, P. H. \& Vogel, H. J. (1955). Amino acid biosynthesis in Torulopsis utilis and Neurospora crassa. J. biol. Chem. 213, 355.

Bartnicki-Garcia, S. \& Nickerson, W. J. (1959). The yeast-like form of Mucor rouxii. Proc. 9th Int. Bot. Cong., Montreal, p. 22.

BeLl, E. T. (1940). The Development of Mathematics, p. 354. New York: McGraw-Hill.

Falcone, G. \& Nickerson, W. J. (1958). Physiological bases of morphogenesis in fungi. 6. Effect of sulfur-deficiency on growth and cellular division of Candida albicans. G. Microbiol. 4, 105.

Falcone, G. \& Nickerson, W. J. (1959). Enzymatic reactions involved in cellular division of microorganisms, in vol. 6, Biochemistry of Morphogenesis. Proc. 4th int. Cong. Biochem., Vienna, p. 65. London: Pergamon Press.

Fruton, J. S. \& Simmonds, S. (1958). General Biochemistry, 2nd ed. New York: John Wiley and Sons, Inc.

Hennessy, D. J. \& Cerecedo, L. R. (1939). The determination of free and phosphorylated thiamine by a modified thiochrome assay. J. Amer. chem. Soc. 61, 179.

Lodder, J. \& Kreger-van RiJ, N. J. W. (1952). The Yeasts, A Taxonomic Study. Amsterdam: North Holland Pub. Co.

Meister, A. (1952). Enzymatic preparation of $\alpha$-keto acids. J. biol. Chem. 197, 309.

Nickerson, W. J. (1954). An enzymatic locus participating in cellular division of a yeast. J. gen. Physiol. 37, 483.

Nickerson, W. J. (1961). Role of biotin in the multiplication of yeasts. Bact. Proc. p. 177.

Radó, T. (1951). On the Problem of Plateau. New York: Chelsea Publishing Co.

Roberts, R. B. \& Abelson, P. H. (1953). The role of the tricarboxylic acid cycle in amino acid synthesis in Escherichia coli. Science, 117, 471.

Rose, W. O. \& Wixom, R. L. (1955). The amino acid requirements of man. XIII. The sparing effect of cystine on the methionine requirement. J. biol. Chem. 216, 763.

Schachner, J. (1929). Trigonopsis variabilis nov.gen. et spec. Z. ges. Braur. 52, 137.

SentheShanmuganathan, S. \& Nickerson, W. J. $(1962 b)$. Composition of cells and cell walls of triangular and ellipsoidal forms of Trigonopsis variabilis. J. gen. Microbiol. 27, 451.

SentheShanmuganathan, S. \& Nickerson, W. J. (1962c). Transaminase and D-amino acid oxidase of Trigonopsis variabilis. J. gen. Microbiol. 27, 465.

Stekol, J. A., Weiss, S., Smith, P. \& Weiss, K. (1953). The synthesis of choline and creatine in rats under various dietary conditions. J. biol. Chem. 201, 299.

Williams, R. R., Waterman, R. E., Keresztesy, J. C. \& Buchman, E. R. (1935). Preparation of pyrimidine and thiazole moieties of thiamin. J. Amer. chem. Soc. 57, 536.

Worwood, A. J. (1949). A method for the estimation of micro amounts of amino nitrogen and its application to paper partition chromatography. Biochem. J. 45, 412.

\section{EXPLANATION OF PLATE}

\section{Plate 1}

Photomicrographs of Trignonopsis variabilis. Outline shown in relief against nigrosin. Both types from liquid cultures incubated for $72 \mathrm{hr}$. at $28^{\circ}$.

Fig. 1. Ellipsoidal organisms grown in medium $\mathbf{A}$, with ammonium sulphate as nitrogen source.

Fig. 2. Triangular forms grown in medium $M$ with DL-methionine as nitrogen source. 